\title{
Versorgungsforschung mit Routinedaten in der Schweiz: eine Herausforderung
}

\section{Claudia Berlin, \\ Radoslaw Panczak, Matthias Egger}

Institut für Sozial- und Präventivmedizin, Universität Bern
Korrespondenz:

Prof. Dr. med. Matthias Egger Institut für Sozial- und

Präventivmedizin (ISPM)

Universität Bern

Finkenhubelweg 11

CH-3012 Bern

Tel. 0316313511

Fax 0316313520

egger[at]ispm.unibe.ch
Medizinische Routinedaten und die auf diesen Daten beruhende Versorgungsforschung sind wichtig als Entscheidungsgrundlage in der Gesundheitspolitik. Studien zu regionalen Unterschieden in der Versorgung spielen eine wichtige Rolle: Ziel ist es, diese Unterschiede zwischen den Regionen zu verstehen und bei ungerechtfertigten Variationen Reformprozesse anzuschieben, um sie zu reduzieren. Warum werden laut medizinischer Statistik der Krankenhäuser zum Beispiel Herzkatheter-Untersuchungen im Kanton Bern doppelt so häufig durchgeführt wie im Kanton Waadt (Abb. 1 nächste Seite) [1]? Handelt es sich um Überversorgung im Kanton Bern oder um Unterversorgung im Kanton Waadt? Werden die Untersuchungen im Kanton Waadt häufiger ambulant durchgeführt? Ist der Bedarf aufgrund des Gesundheitszustands der Bevölkerung im Kanton Waadt tiefer, oder besteht vielleicht im Kanton Bern eine angebotsinduzierte Nachfrage? Solche Fragen können nur mit Hilfe von kleinräumig organisierten und verknüpfbaren Routinedaten sowie vertiefenden Erhebungen beantwortet werden.

Public Health Schweiz forderte letztes Jahr in einem Manifest «Bessere Gesundheitsdaten für ein effizienteres Gesundheitswesen» [2]: Vollständigkeit und Verfügbarkeit, aber auch die Verknüpfbarkeit und Vergleichbarkeit der Daten sind unbefriedigend. Hier stellen wir wichtige Datenquellen vor und berichten über unsere Erfahrungen mit deren Verwendung in der Versorgungsforschung.

\section{Verknüpfung von Datenbanken}

Eine Verknüpfung von Datensätzen kann mit Hilfe einer eineindeutigen Identifikationsnummer (zum Beispiel die «Personennummer» in Schweden) oder mittels Record-Linkage unter Verwendung mehrerer Variablen vorgenommen werden (z.B. Geburtsdatum, Wohnort, Geschlecht, Zivilstand, Nationalität zur Verknüpfung von Volkszählungs- und Sterbedaten in der Swiss National Cohort [3]). Ist die Verknüpfung auf Ebene Individuum nicht möglich, dann können die Daten mit Hilfe von räumlichen Informationen verknüpft werden. So kann die Prävalenz einer Krankheit auf Gemeindeebene abgebildet werden, indem die Daten zur Krankheitshäufigkeit mit der Bevölkerungsstatistik in Beziehung gesetzt wird. Die Verknüpfung wird erschwert, wenn die Datensätze aufgrund unterschiedlicher geographischer Bezugsgrössen erstellt wurden, die nicht oder nur

\section{Die Identifikation und Untersuchung von regionalen} Unterschieden bei der medizinischen Versorgung in der Schweiz ist ein Schwerpunkt der medizinischen Versorgungsforschung. Eine evidenzbasierte Versorgungsforschung erfordert medizinische Routinedaten, die die Anforderungen der Vollständigkeit, Verfügbarkeit, Verknüpfbarkeit und Vergleichbarkeit erfüllen. Die vorhandenen medizinischen Routinedaten kommen diesen Anforderungen teilweise nur ungenügend nach («Bessere Gesundheitsdaten für ein effizienteres Gesundheitswesen», Public Health Schweiz). In diesem Artikel berichten wir über eine Auswahl wichtiger Datenquellen und den Herausforderungen, denen wir bei der Verknüpfung verschiedener Datensätze und der Auswertung gegenüberstanden.

teilweise miteinander kompatibel sind (z. B. Postleitzahl-Bereiche und Gemeinden). In diesem Fall muss eine grössere geographische Einheit gewählt werden, z.B. der Kanton (Abb. 1). Grundsätzlich sollte die kleinste mögliche Einheit verknüpft werden. Eine Auswertung mit grösseren Einheiten ist dann immer noch möglich.

Wenn Daten im Rahmen von Zeitreihenuntersuchungen ausgewertet werden, können sich weitere Schwierigkeiten ergeben. Postleitzahl-Gebiete sowie Gemeindegrenzen, -namen und -nummern können sich im Laufe der Zeit ändern. Gemeinden können fusionieren, werden geteilt oder an andere Gemeinden angegliedert. Wenn solche Änderungen in mehreren Datensätzen mit unterschiedlichen zeitlichen und räumlichen Bezugseinheiten auftreten, ist die Verknüpfung aufwendig und es wird schwierig, ein stabiles Model für die räumliche Analyse aufzubauen.

\section{Datensätze im stationären Sektor}

Alle Schweizer Spitäler sind verpflichtet, Daten über die behandelten Patienten sowie die personelle und technische Infrastruktur des Krankenhauses zu erheben. Daraus ergeben sich Datensätze, die das Bundes- 


\section{Abbildung 1}

Standardisierte Raten von Herzkatheter-Untersuchungen nach Kanton im Jahr 2011 (per 100 000).

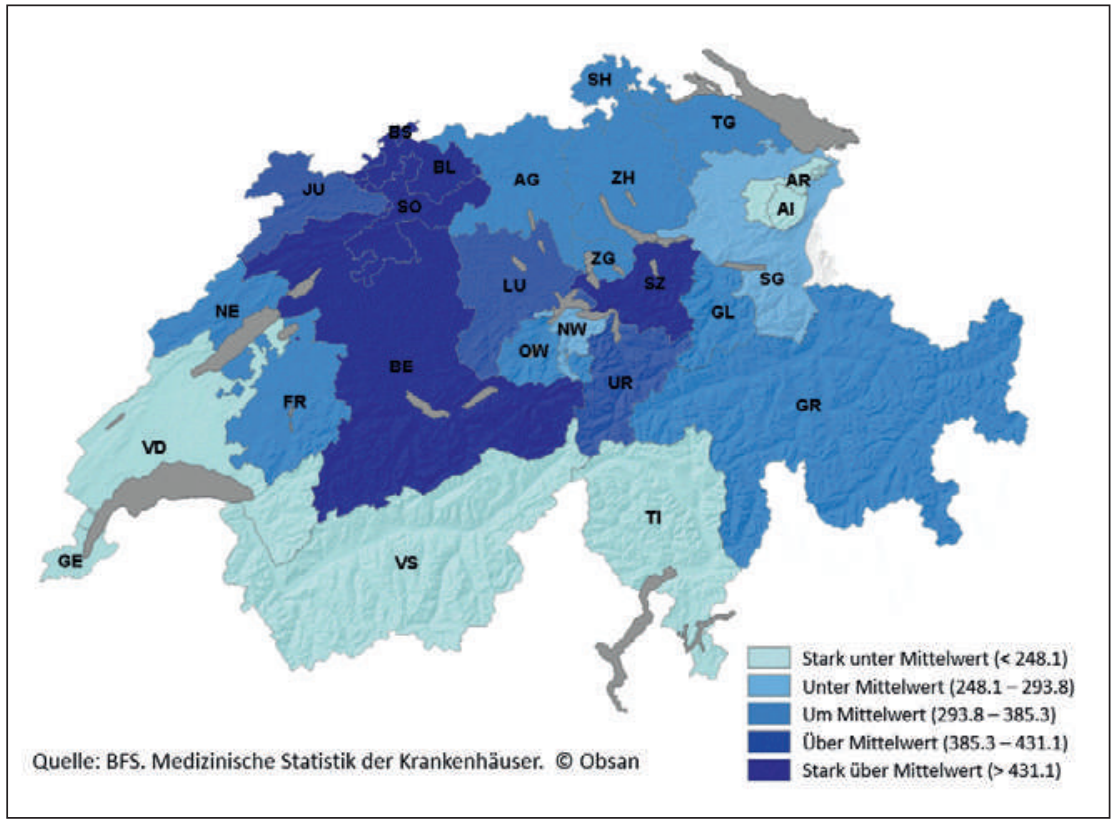

amt für Statistik (BFS) aufbereitet und für die Analyse der stationären Versorgung zur Verfügung stellt:

- die Krankenhausstatistik

- die Medizinische Statistik der Krankenhäuser

Die Medizinische Statistik der Krankenhäuser (MS) enthält Angaben zu allen stationär behandelten Krankheitsfällen in den Schweizer Spitälern. Bei jedem Fall wird eine Vielzahl an Variablen erhoben (Tab. 1). Die Krankenhausstatistik (KS) enthält Daten zur Infrastruktur der Spitäler. Hierzu gehören z. B. die Anzahl der Betten, die Anzahl der Magnetresonanztomographiegeräte, oder die Anzahl der Beschäftigten. KS und MS können über die Spital-ID miteinander verknüpft werden. Um die Anonymität der Patienten zu gewährleisten, wurden 706 sogenannte Medstat-Regionen eingeführt. Hiermit werden Angaben zum Wohnort des Patienten und zum Standort des Spitals kodiert. Dabei bilden mehrere Postleitzahl-Gebiete eine Medstat-Region. Das BFS veröffentlicht jedes Jahr eine neue Postleitzahl-Medstat-Transkodiertabelle, da es bei den Postleitzahlen (PLZ) häufig zu Änderungen kommt.

\section{Zürcher Patienten in Schaffhauser Spitälern}

In unserer Forschungsgruppe werden die MS und KS der Jahre 2008 bis 2011 im Rahmen verschiedener Projekte verwendet, um die allgemeine und die krankheitsspezifische stationäre Versorgung in der Schweiz abzubilden. Zur Verortung im Raum sowie zur Darstellung von Versorgungsstrukturen verwenden wir die Medstat-Regionen. Plausibilitätsuntersu- chungen liessen uns jedoch bald stutzig werden. Beim Vergleich der Fallzahlen je Medstat-Region traten zum Teil massive Schwankungen von Jahr zu Jahr auf. Ausserdem zeigten sich sehr ungewöhnliche Behandlungspfade. So wurde z.B. die Hälfte der PatientInnen, die im Jahr 2008 in der MedstatRegion ZH52 (östlich des Zürichsees) lebte, in den Spitälern Schaffhausens behandelt. Im Jahr 2011 waren es hingegen nur 2 Patient(inn)en.

Es stellte sich heraus, dass dies mit einer Überarbeitung der Medstat-Regionen zusammenhing, die zur Jahreswende 2007/2008 durchgeführt wurde. Damals wurden viele Medstat-Regionen neu zusammengesetzt, um die Medstat-Regionen mit den Arbeitsmarkt-Regionen (MS-Regionen, «Mobilité Spatiale») kompatibel $\mathrm{zu}$ machen. Allerdings wurden viele Medstat-Bezeichnungen beibehalten, obwohl diese nun teilweise andere Gegenden in den Kantonen benannten. So bezeichnete die in unserem Beispiel genannte Medstat-Region ZH52 im Jahr 2007 ein Gebiet südlich der Grenzen zum Kanton Schaffhausen, ab 2008 wurde damit eine Region östlich des Zürichsees benannt. Weitere Recherchen ergaben, dass das Software-Update, das die neue Transkodiertabelle enthielt, von einer der IT-Firmen erst in den Jahren 2009 oder 2010 an die Spitäler versandt wurde. Die Spitäler, die das Krankenhausinformationssystem dieser Firma verwendeten, griffen somit auf die alte Transkodiertabelle zu und wiesen daher ihre PatientInnen falschen Regionen zu.

Tabelle 1
Einige der Variablen, die bei einem stationär behandelten
Krankheitsfall erhoben werden.
- Fall-ID
- Patienten-ID
- Spital-ID
- Geschlecht
- Alter
- Wohnregion des Patienten
- Standort des Spitals
- Eintrittstag
- Austrittstag
- Eintrittsart (z.B. Notfall)
- Austrittsart (z.B. Tod, Überweisung)
- Hauptdiagnose
- Nebendiagnosen
- CHOP-Codes*
- APDRG** (ab 2012 SwissDRG)
* Bei den CHOP-Kodes handelt es sich um Behandlungskodes
(Schweizerische Operationsklassifikation).
** APDRG: «All Patient Diagnosis Related Groups». Dieses
Fallpauschalensystem wurde in einigen Kantonen zur
Abgeltung akutsomatischer Spitalbehandlungen angewandt.
2012 wurde es vom schweizweit geltenden SwissDRG
abgelöst.


Mehr hospitalisierte Patienten als Einwohner

In einer Medstat-Region östlich von Winterthur überstieg die Anzahl der hospitalisierten Fälle die Anzahl der in dieser Region lebenden Personen. Es stellte sich heraus, dass die PLZ, die zur Zuordnung der Patienten zu einer Medstat-Region verwendet wurde, nicht der tatsächlichen PLZ des Patienten entsprach. In vielen Gemeinden existieren mehrere Postleitzahlen oder PLZ gelten für mehrere Gemeinden. So wurde für die Zuordnung der Winterthurer Patienten zur Medstat-Region eine PLZ benutzt, die sowohl für die Stadt Winterthur als auch für eine Gemeinde östlich der Stadt gültig war. Die Patienten wurden also in einem ersten Schritt zwar der korrekten Gemeinde (Stadt Winterthur) zugeordnet. Im zweiten Schritt wurde aber eine PLZ verwendet, die auch für eine Gemeinde ausserhalb der Stadt Winterthur benutzt wird. Die Transkodiertabelle ordnete diese PLZ dann der Medstat-Region östlich von Winterthur zu.

\section{Verschwindende Spitäler und andere Zuordnungsprobleme}

Durch das Fusionieren von Spitälern wurden die Daten der einzelnen Spitäler nur noch über eine einzige Spital-ID übermittelt, obwohl zum neuen Spitalverbund Spitäler an verschiedenen Standorten gehörten. Eine Zuordnung der Patient(inn)en zur korrekten Spital-Medstat-Region wurde dadurch verunmöglicht.

Patient(inn)en, die mehrfach hospitalisiert wurden, können normalerweise anhand einer anonymisierten Patienten-ID identifiziert werden. Zur Erzeugung der Patienten-ID werden Vorname, Familienname, Geburtsdatum und Geschlecht verwendet. Diese Daten werden dann mit Hilfe eines sogenannten «Hash»-Codes anonymisiert. Allerdings sind die auf diese Weise konstruierten IDs nicht eineindeutig. Probleme treten bei Änderungen des Familiennamens auf, da dann eine andere ID konstruiert wird, obwohl es sich um die gleiche Person handelt.

\section{Zweimal gestorben}

Die Statistik der Sozialmedizinischen Institutionen (SOMED) enthält Angaben zu allen sozialmedizinischen Einrichtungen der Schweiz. Im Rahmen eines Projektes zur Versorgung im letzten Lebensjahr verknüpften wir anhand der anonymisierten Patienten-ID die MS- und SOMED-Daten verstorbener Patient(inn)en. Wir stellten fest, dass einige Personen sowohl in der MS als auch in der SOMED als verstorben kodiert wurden, obwohl der Tod der Patient(inn)en nur in einer der Einrichtungen eingetreten sein kann. Mögliche Gründe dafür sind nicht eindeutige PatientenIDs und Kodierfehler. Weitere Probleme bereiten überlappende Aufenthalte in verschiedenen Einrichtungen. Diese kommen zustande, wenn Betten von überwiesenen PatientInnen bis zu ihrer Rückkehr freigehalten werden. Dies führt dazu, dass die tat- sächliche Behandlungsdauer in einer Institution nicht eindeutig zu bestimmen ist.

\section{Fehlende Auflösung und falsche Wohnorte}

Für die Versorgungsforschung sind exakte Angaben zur Anzahl, Spezialisierung und Inanspruchnahme von medizinischen Leistungserbringern unerlässlich. In der $K S$ geben Spitäler mit mehreren Aktivitätstypen (z. B. Akutversorgung, Psychiatrie, Geriatrie) jedoch nur die Gesamtzahl ihrer Betten an: die Betten werden nicht nach den Fachabteilungen aufgeschlüsselt.

Im ambulanten Bereich können die Zahlstellenregister-Nummern (ZSR-Nummern) der zugelassenen medizinischen Leistungserbringer nicht immer einem einzelnen Arzt zugeordnet werden. Unter einer ZSR-Nummer kann auch eine ganze Gruppenpraxis abrechnen.

Schliesslich mussten wir feststellen, dass die Variable Wohnort in der MS-Statistik im Zeitraum 2008-2010 oft fehlerhaft war. Von kleinräumigen Analysen anhand der Medstat-Regionen ist deshalb abzuraten. Analysen, die mit diesen Daten durchgeführt wurden (z. B. im Rahmen von kantonalen Versorgungsplanungen), sollten kritisch hinterfragt werden.

\section{Lösungsansätze}

Die Zuordnung der Medstat-Regionen erfolgt durch die Leistungserbringer bzw. Kantone und ist somit für das BFS nicht nachvollziehbar oder überprüfbar. Das BFS hat die Spitäler und Kantone darauf hingewiesen, dass bei der Zuordnung zu Medstat-Regionen die korrekte Transkodiertabelle zu verwenden ist. Der Problematik der Spitäler-Fusion versucht das BFS seit 2011 durch die Einführung einer neuen Variablen «Spitalstandort» Herr zu werden.

Wir unterstützen die von Public Health Schweiz formulierte Forderung nach einer einheitlichen, anonymisierten Identifikationsnummer [2]. Diese soll für alle medizinischen Datensätze gelten, um die Verknüpfbarkeit zu verbessern. Ein weiterer Lösungsansatz ist die Verknüpfung mit Hilfe von geokodierten Adressen, d. h. die Zuordnung der jeweiligen geographischen Koordinaten zu einer Adresse. Dadurch ist eine präzise Ortsangabe möglich und die Zuweisung einer administrativen bzw. geographischen Einheit zu den erhobenen Daten ist einfach. In der Schweiz sind die entsprechenden Datengrundlagen vorhanden (z.B. Gebäudekoordinaten vom Bundesamt für Landestopografie Swisstopo). Selbstverständlich müsste die Geokodierung der Patienten- und Leistungserbringer-Adressen äusserst strengen Datenschutzbestimmungen unterliegen. Gleiches gilt auch für die Aufbewahrung der Adresskoordinaten. Dies könnte dem im Manifest [2] vorgestellten Trust Center übertragen werden, dessen Aufgabe auch die Sicherung der einheitlichen Identifikationsnummern ist. Werden für eine Analyse die geographi- 
schen Informationen zu einem Patienten oder zu einem Leistungserbringer benötigt, kann das Trust Center die Adresskoordinaten einfach der benötigten geographischen Einheit (z.B. Gemeinde) zuweisen und diese dann übermitteln, ohne dabei die Koordinaten herauszugeben.

Die Medstat-Regionen werden in der MS und KS angewendet und sollen auch bei der Erhebung von Daten zum ambulanten Gesundheitssystem der Schweiz (MARS-Projekt: Modules Ambulatoires des Relevés sur la Santê) zum Einsatz kommen. Wir schlagen

\section{Dialoggruppe Forschungsschwerpunkt Versorgungsforschung}

Versorgungsforschung ist für die Ärzteschaft ein wichtiger und wegweisender Wissenschaftsbereich. In Zeiten des Umbruchs und der Veränderungen im Gesundheitswesen (neue Finanzierungsmodelle, demographische Veränderungen, steigende Gesundheitskosten usw.) ist eine akademisch verankerte Forschung im Bereich der ärztlichen Versorgung zwingend nötig. Um wissenschaftliche, von Partikulärinteressen unabhängige Grundlagen schaffen zu können, unterstützen die Verbindung der Schweizer Ärztinnen und Ärzte (FMH), die Konferenz der Kantonalen Ärztegesellschaften (KKA) sowie Newlndex gemeinsam den Forschungsschwerpunkt Versorgungsforschung am Institut für Sozial- und Präventivmedizin der Universität Bern.

Eine Dialoggruppe dient als Informations- und Austauschplattform: Vertreter der genannten Organisationen und der Forschungsgruppen, diskutieren regelmässig die laufenden und geplanten Arbeiten im Bereich der Versorgungsforschung. Die Dialoggruppe verfolgt zudem das Ziel, die Akzeptanz und Sensibilisierung innerhalb der Ärzteschaft für diesen Wissenschaftsbereich zu fördern.

Die Dialoggruppe steht ihrer Basis offen für Themen-, Diskussionsvorschläge sowie für weitere Fragen und Informationen. Die Abteilung Daten, Demographie und Qualität DDQ der FMH übernimmt die Koordination der Dialoggruppe und steht für weitere Informationen und Auskünfte gerne zur Verfügung: ddq[at]fmh.ch oder Tel. 0313591111. vor, stattdessen die Adressen zu geokodieren. Auf diese Weise kann die Zuverlässigkeit der geographischen Variablen und damit auch der Datenauswertung deutlich verbessert werden.

\section{Fazit und Ausblick}

Die hier aufgeführten Beispiele bilden nur einen Teil der für die Versorgungsforschung wichtigen Datensätze. Erfahrungsgemäss werden Probleme meist erst durch die Verwendung und Analyse der Daten sichtbar. Gesammelte Daten sollen regelmässig analysiert werden, damit die Daten verbessert und Eingang in die aktuellen Entscheidungsprozesse im Gesundheitswesen finden können. Die beschriebenen Probleme könnten relativ einfach gelöst werden. Ob der politische Wille dazu vorhanden ist, wird die $\mathrm{Zu}$ kunft zeigen. Die Entwicklungen im Bereich der Krebsregistrierung geben aber Anlass zur Hoffnung, dass eine fundierte und breitabgestützte Versorgungsforschung in einigen Jahren auch in der Schweiz möglich wird.

\section{Literatur}

1 Pellegrini S, Kohler D, Otto S. Variations géographiques dans les soins de santé. La situation en Suisse. Un projet de l'OCDE. Obsan: Neuchâtel; 2014.

2 Public Health Schweiz. Manifest. Bessere Gesundheitsdaten für ein effizienteres Gesundheitswesen. Erhältlich unter www.hausarztmedizin.uzh.ch/ aktuell/130816-ManifestGesundheitsdaten-D-def.pdf (accessed 2 Nov 2014).

3 Bopp M, Spoerri A, Zwahlen M, Gutzwiller F, Paccaud F, Braun-Fahrländer C, et al. Cohort Profile: the Swiss National Cohort--a longitudinal study of 6.8 million people. Int J Epidemiol. 2009;38:379-84. 